\title{
DESIGN OF QUICK CLAMPING DEVICE OF PERIPHERAL ATTACHMENTS FOR SKID-STEER LOADER
}

\author{
Miroslav Blatnicky, Jan Dizo \\ University of Zilina, Slovakia \\ miroslav.blatnicky@fstroj.uniza.sk,jan.dizo@fstroj.uniza.sk
}

\begin{abstract}
The objective of the article is to present a design and optimisation of a quick clamping device for changing peripheral attachments, which is intended to be mounted on a skid-steer loader Locust produced in the Slovak Republic. It is the only producer of skid-steer loaders in the Slovak Republic, which parameters, such as operating weight, tipping load, breakout force, engine power, hydraulic flow, travel speed etc. compete with world producers. In this article, the design, drive-train and control system of the skid-steer loader are described, as well as the presentation of a current state of the quick clamping device is introduced, which is modified and optimised based on the producer requirements. The modification of the quick clamping device is necessary due to the simultaneous manual actuating of the attachments locking. A loader operator has to get in the loader cabin, to hang up an attachment on the quick clamping device, then to get off the cabin and manually lock the attachment by means of a lever system. After this process he can get in the cabin and use the loader. The modification of the quick clamping device will allow to lock the peripheral attachments directly from the loader cabin. It will improve comfort of an operator, while guaranteeing safety of the loader operation. The most important objectives of the presented technical solution were to meet all producer's requirements together with the best functionality, in order to implement the designed solution to the current manufacturing process of the company. The numerical analysis of the quick clamping device design is included in the article. The boundary conditions needed to the numerical calculation have been determined based on maximal load capacity of used hydraulic cylinders ensuring proper kinematics of the mechanism.
\end{abstract}

Keywords: skid-steer loader, attachments, solution, analysis.

\section{Introduction}

In the past, all manual work was done by hand, and for that reason it took a long time to do everything. In this day and age, however, we know a large number of different types of ground handling machines. The largest of these are giant crawler excavators used in coal mines. These excavators are exceptional, because their size does not allow them to be used everywhere in the world. On the contrary, this article focuses on the smallest machines in the field of ground handling equipment. These are skid steer loaders. There are two variants, with a classic wheeled chassis or a tracked chassis. Their manoeuvring ensures wheel slip [1-3].

This type of control significantly reduces the turning radius, which is a great advantage in small work areas. Thanks to their excellent maneuverability, skid steer loaders are popular in the construction and agricultural sectors. Their small design gives them an advantage over large loaders. Sliding wheel drives ensure a low turning radius, which they use when handling material in narrow storage areas. They are used for transport of palletized material, also for handling of loose materials, during excavation work, or for cleaning and treatment of urban public areas. They are the most compact loaders available on the market, thanks to the wide choice of working tools (peripherals), such as a tooth shovel, pallet forks, a sweeper etc. [4-6].

The basic design of the loader (Fig. 1) does not vary greatly from one manufacturer to another. The rigid frame (1) is an integral part of the loader construction. In the lower part of the frame there is an internal combustion engine (2), which together with the pumps $(4,5)$ forms one assembly. The engine also serves as a counterweight to the loader. A combined cooler (6) is installed downstream of the engine to cool water and working fluid. The fuel tank (7) is located on the right side of the engine outside the frame. The hydraulic tank (8), in which the working fluid filter is located, is on the left side of the engine outside the frame. The hydraulic distributor (9) is located on the lower right side of the frame. The driver's working cab (10) is mounted on the frame and can be folded out. It contains all the controls and the driver's seat.

The entrance door serves as a viewing window. The cab, together with all the elements, forms another assembly. The boom loader (11), to which the working tool (12) is attached, is mounted by means of pins in a rigid frame. The wheels (13) are mounted on the side transmissions (14) at the sides of the frame. The water heater (15) is on the outside of the cab. The accumulator (16) is on the right 
side of the frame and can be accessed by tilting the cab and covers with the boom raised. The beacon (17) is magnetic and, in use, connects to the wiring socket.

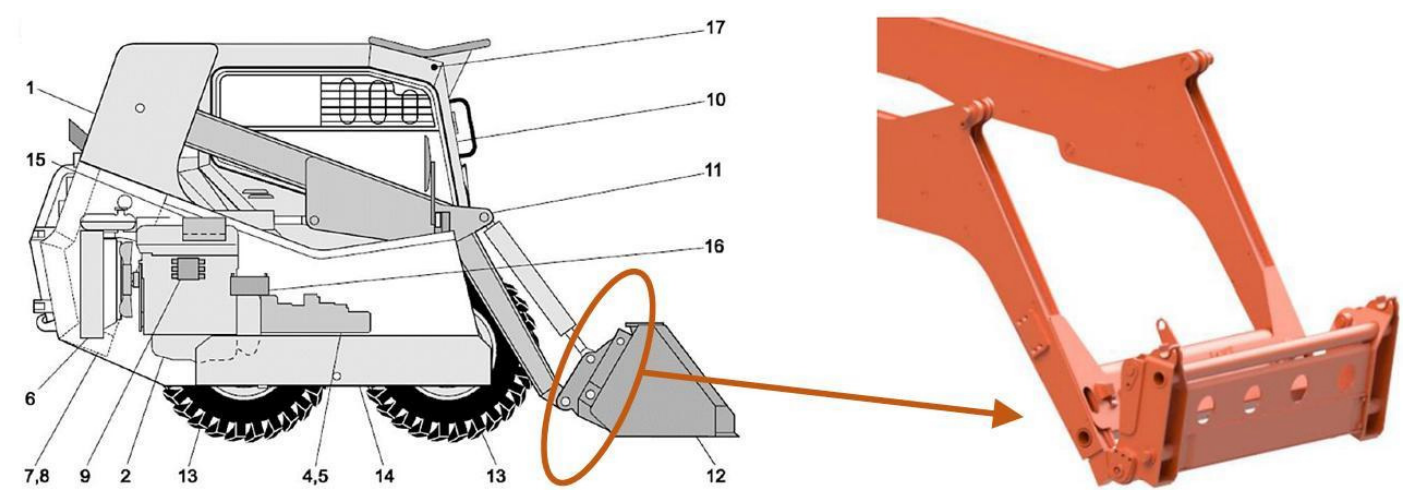

Fig. 1. Basic assembly of the Locust L 1203 (left) and the part of the solved loader design (right)

\section{Materials and methods}

The design of the quick clamping device of the particular solved skid-steer loader has been performed based on using of analytical and numerical methods, which are described below.

The Locust combined cooler (Fig. 2) is connected to the water pump via the lower chamber. The upper chamber of the cooler is connected by a hose to the neck of the liquid outlet from the engine in which the thermostat is located. On the cooler there is a balancing tank with a pouring neck, closed by a pressure plug. The lower chamber has a drain cock. The hinged cooler provides good access to the engine compartment for maintenance and cleaning (Fig. 2).

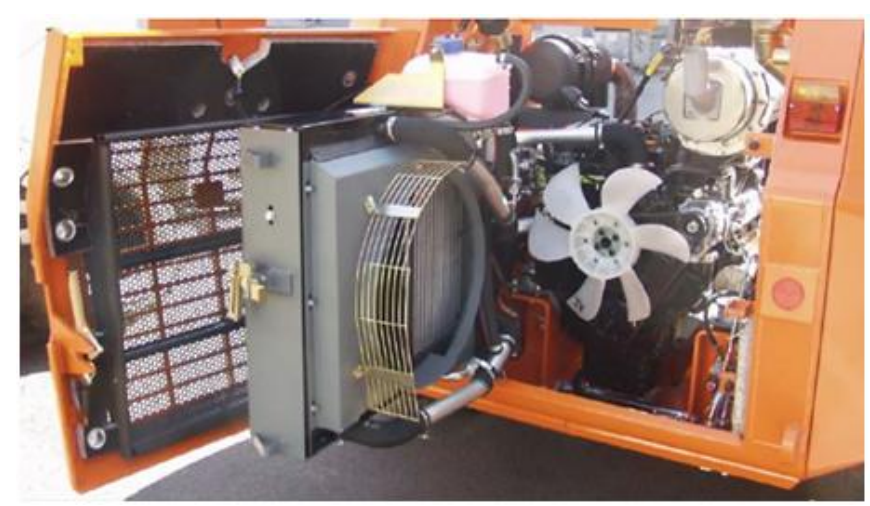

Fig. 2. View of the combined cooler open

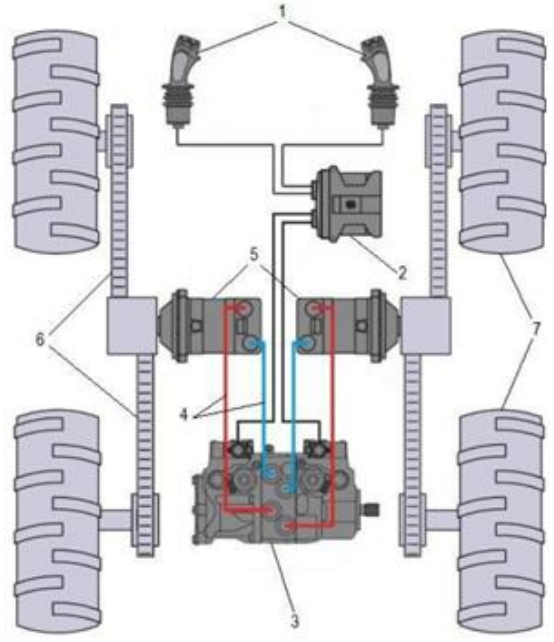

Fig. 3. Basic scheme of the skidsteer drive system

Each side of the loader drive has a separate hydraulic generator and hydraulic motor (Fig. 3). The control levers (1) located in the driver's cab are used to control the drive. The hydraulic generator (2) is driven by an internal combustion engine (3) via a drive housing and supplies hydraulic fluid (4) to the hydraulic motor (5) located between the front and rear wheels. The driving torque is transmitted from the two-pinion of the hydraulic motor to the side wheel gearbox and from there via the chain gear (6) to the front and rear wheels (7). All wheels are thus driven. The entire scheme is shown in Fig. 3.

With the same forward movement of the levers, the same amount of hydraulic fluid flows into both pumps. The hydraulic motors have the same revolutions, so that the wheels move at the same speed and the vehicle points straight ahead. By shifting one of the levers less, we change the direction of the loader, because the speed in one of the hydraulic motors decreases and as a result the loader starts to turn. When one lever is moved forward and the other lever backwards, the wheels turn in the opposite direction as those on the other side, starting to rotate the vehicle in place [7-10]. For some 
manufacturers, the loader is operated with only one lever, while the other is used to manipulate the boom and the implement. The loader drive is hydrostatic. The drive unit is an engine, which power gives the loader good dynamic performance. The engine torque is transmitted to the pumps by the flywheel and clutch. The clutch is fixed, non-disengageable. The engine is fitted with a Bosch tandem pump, which consists of two control pumps to drive and control the axial pump, which feeds the hydraulic system of the working equipment. From the control pumps, the torque is transmitted hydrostatically to the MCR 5 hydraulic motors by means of high-pressure hoses. The MCR 5 hydraulic motors are fitted with wet multi-plate friction brakes, which by their design ensure the braking of the skid-steer loader, serve as emergency and parking brakes.

The quick clamping device is an important part of the loader construction. It is mounted at the end of the boom and serves as a link between the loader and the implement. Thanks to the quick clamping device, it is possible to change the work tools in a few minutes, without the need for lengthy mounting to the loader boom. The current clamping device mounted on the boom is shown in Fig. 4.

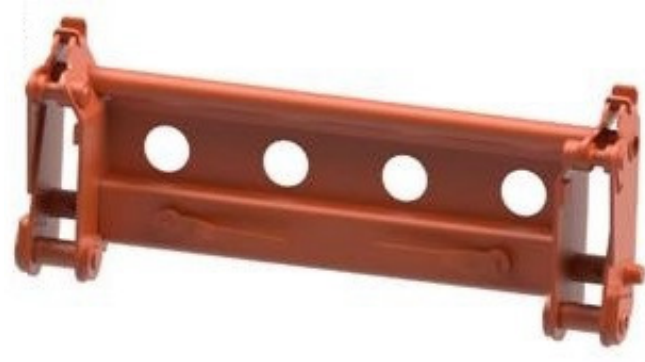

Fig. 4. Quick clamping device, the current design

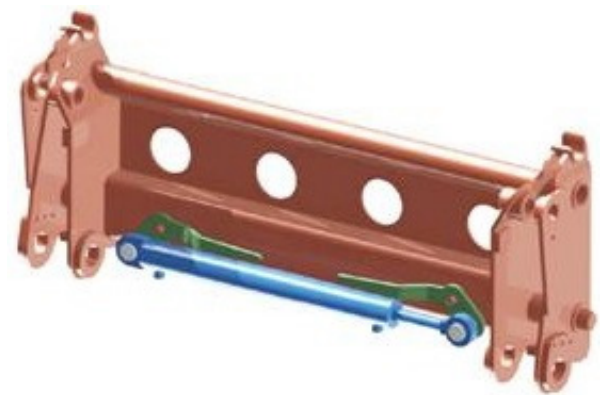

Fig. 5. Quick clamping device, the proposed design modification

The main parts of the quick clamping device are two side frames, which are dimensionally identical, but mirror-inverted. They are the supporting parts of the entire quick clamping device structure. At the top, they have metal cases for storing work tools. Locust models use a quick clamping device with manual locking of the implement to the boom. The principle of this locking lies in two pins that slide out of the quick clamping device construction into the holes on the tool and prevent its movement in directions perpendicular to the axis of the pins. Extending the pins is ensured by the movement of the levers. Each pin is operated by one lever. This means that the pins can move independently from each other. The entire structure of the quick clamping device is shown in Fig. 4.

The main disadvantage of the current solution is the manual control of the working tool securing. The operator of the loader has to get into the cab, hang the working tools on the quick clamping device and then step out of the loader and manually secure the working tools using the levers. After this process, he can re-enter the cab and use the tool safely. The WAY Industries' requirement is to eliminate this lengthy process of securing and unlocking work tools. The main objective is to allow the loader operator to conveniently operate the locking system from the loader cab. This will reduce the time required to secure the implement and at the same time increase the safety of the loader, as they will not have to handle the unsecured parts of the loader.

Another requirement is to keep the basic dimensions of the current quick clamping device design. There must be no displacement of the side frames of the quick clamping device or the cross tube connecting them. It is necessary to maintain the distance between these components, as they have brackets for attachment to the boom. Modifications are only permitted on the cross member and quick clamping device components or attachments.

The basic part of the design is a double-acting hydraulic cylinder shown in Fig. 5 on the right. The hydraulic cylinder is attached to the quick clamping device structure by means of pins. The compressive force is transferred from the hydraulic cylinder by means of the pins to the pivot levers that extend or retract the pins securing the implement. When selecting a hydraulic cylinder, the main criteria were its basic dimensions. The most important parameters were the total length of the cylinder and the piston stroke. The axes of the holes in the pivoting levers, into which the pins are inserted, are spaced $a=656.24 \mathrm{~mm}$ apart. The length of the end parts of the pins for securing the tool is in the 
extended state $l=1082.8 \mathrm{~mm} \quad$ (Fig. 6). After inserting the pins, the length is shortened to $l^{\prime}=1026 \mathrm{~mm}$. The required piston stroke $z$ will then be as following:

$$
z=l_{1}-l_{2} \text {, }
$$

where $l_{1}$ - length of the end parts of the pins in the extended state, $\mathrm{mm}$;

$l_{2}-$ shortened length due to inserting pins, $\mathrm{mm}$.

Substituting numerical values to eq. (1) gives:

$$
z=1082.8-1026=56.8 \mathrm{~mm} .
$$

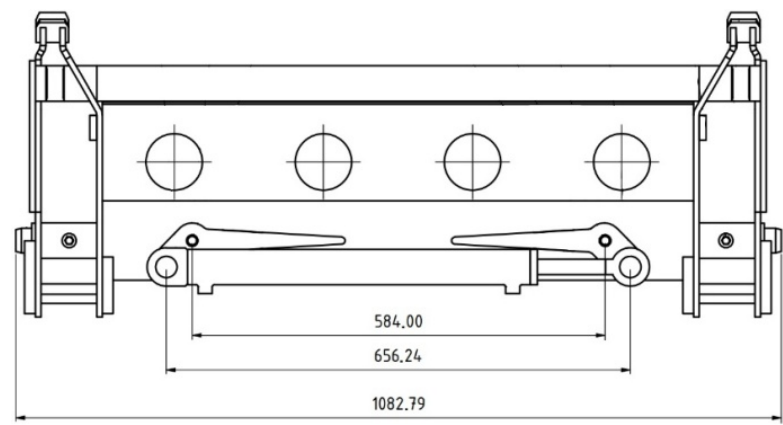

Fig. 6. Geometry of the selected double-acting hydraulic cylinder

It follows from equation (2) that the necessary stroke of the hydraulic cylinder piston is of 56.8 $\mathrm{mm}$. Of the available sources, Hydrolider was the most suitable hydraulic cylinder for these dimensions. The entire designation of the hydraulic cylinder is CJ2F 40/22/350 U25. The basic dimensions of the selected hydraulic cylinder are shown in Fig. 7.

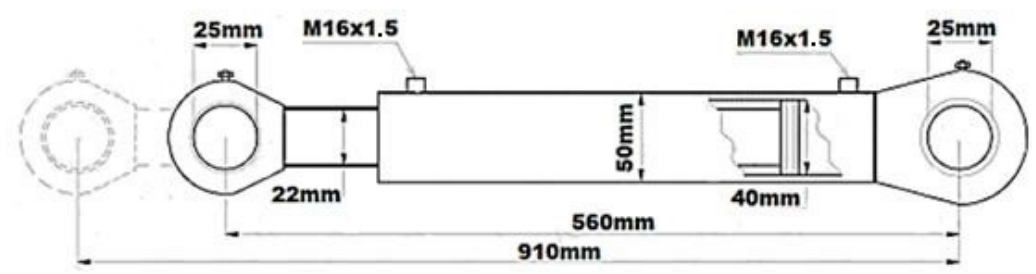

Fig. 7. Geometry of the selected double-acting hydraulic cylinder

The maximum hydraulic fluid pressure $p_{\max }$ in the working cycle is of $18.5 \mathrm{MPa}$. This pressure applies to the hydraulic cylinders controlling the boom movement and tilting of the implement. On the boom there are two other hydraulic hoses ended with quick - acting couplings marked FFI 34 GA M (Fig. 7). The pressure in these hoses is the same as in the whole working cycle. The hoses are used to supply the hydraulic fluid to the hydraulic motor on the implement, if any. In this design, the hydraulic cylinder is connected to the two hoses. The maximum power of the hydraulic cylinder is calculated from the dimensions of the cylinder and the maximum working pressure at which the cylinder works. Equation (3) expressing the formula for calculating the maximum extension force $F_{\max }$ of the hydraulic cylinder:

$$
F_{\max 1}=\frac{p_{\max } \cdot \pi \cdot D_{1}^{2}}{4} \cdot \eta,
$$

where $F_{\max 1}-$ maximum extension force, N;

$p_{\max }-$ maximum fluid pressure in the working cycle, $\mathrm{MPa}$;

$D_{1}$ - internal diameter of the hydraulic cylinder, $\mathrm{mm}$;

$\eta$ - total mechanical efficiency of the hydraulic cylinder.

The total mechanical efficiency of the cylinder stated by the manufacturer is of $95 \%$.

Then, after substituting the corresponding values in relation (2), we get $F_{\max }=22085.4$ $N$. Equation (3) expressing the formula for calculating the maximum insertion force $F_{\max 2}(N)$ of the hydraulic cylinder: 


$$
F_{\max 2}=\frac{p_{\max } \cdot \pi \cdot\left(D_{1}^{2}-d_{1}^{2}\right)}{4} \cdot \eta,
$$

where $F_{\max 1}-$ maximum extension force, $\mathrm{N}$.

Substituting the respective values into equation (4) we obtain the value of the force $F_{\max 2}$ of $15404.56 \mathrm{~N}$. The calculated maximum forces that the hydraulic cylinder can exert when extending and retracting are too high. In the event that something prevents the locking pins from extending, such a large force could damage the design of the quick clamping device or the tool. Therefore, safety valves installed in the hydraulic system were implemented in the design and were set to a working pressure of $3 \mathrm{MPa}$. Substituting the value of the operating pressure of $3.0 \mathrm{MPa}$ in the circulation into equations (2) and (3), we obtain the value of the extension force $F_{v l}$ of $3581.4 \mathrm{~N}$, and the insertion force $F_{z l}$ of $2498.025 \mathrm{~N}$. Such high forces, as shown by practical experience in the operation of the device, are sufficient to extend and retract the locking pins, while minimizing the risk of damage to the quick clamping device structure and the tool. A hydraulic and electrical diagram was prepared for this design.

The two-position manual switch located in the cab, within the reach of the loader operator, controls the hydraulic cylinder. The switch sends an electrical signal to the trigger of the coil. The coil is used to control the position of the valve located in the hydraulic system and regulates the flow of hydraulic fluid between the hydraulic unit and the hydraulic cylinder. The system also has a pressure regulator set to a pressure $p$ of $3.0 \mathrm{MPa}$.

\section{Results and discussion}

This section contains description of the result design of the quick clamping device and a result of stress analysis of its most loaded part. The result technical solution of the solved quick clamping device includes a double-acting hydraulic cylinder, which is used to change the hydraulic force from the pump to mechanical force with a sliding motion. The pins made of low alloy steel, shown in Fig. 8, are inserted into the sleeves of the hydraulic cylinder. The pins serve to transmit the compressive force produced by the cylinder. The force is transmitted to the pivot levers, in which the narrower part of the pin is mounted. The wider part of the pin has a diameter of $\varnothing 25 \mathrm{~mm}$, the narrower part has a diameter of $\emptyset 15 \mathrm{~mm}$ (Fig. 9).

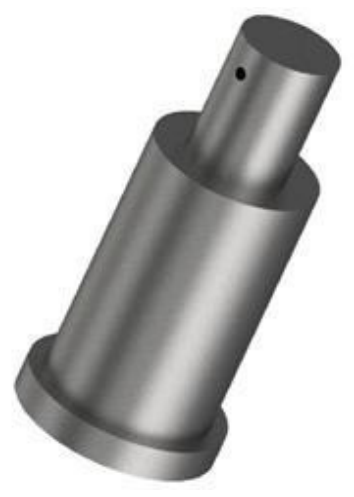

Fig. 8. 3D model of a steel pin

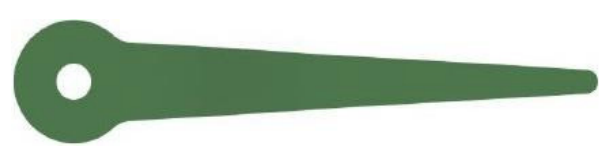

Fig. 10. Original design of a lever

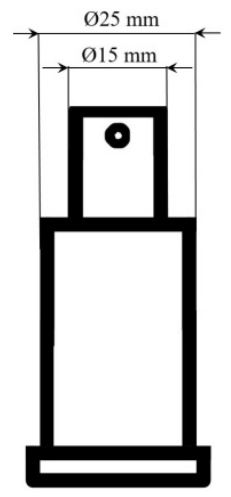

Fig. 9. Main diameters of the pin

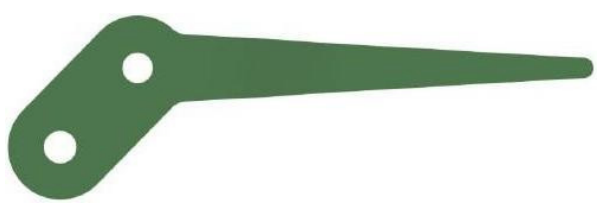

Fig. 11. Modified design of a lever

The pivot levers (Fig. 10, Fig. 11) transfer the force to the pull-out pins, which serve to secure the working tool. The entire layout of the mechanism is shown in Fig. 12. No modifications were necessary to the design of the quick clamping device frame. All dimensions were maintained in its 
original values. The mechanical lever consists of two basic parts. The lever itself and the bushing, which is attached to the lever by weld joint. Adjustments were made only on the main part of the lever. To fix the pin, it was necessary to extend the part of the lever and drill a hole with a diameter of $\varnothing 15 \mathrm{~mm}$ into which the pin will be inserted. A comparison of the original and the new lever arrangement is shown in Fig. 10 on the right. The main advantage of the new design (Fig. 12) is the simple modification of the original locking system. It is only needed to replace the original pivot levers with the new ones and install the new components in the system. Such solution is cost effective and it also reduces the time required to adjust the quick clamping device. In the event of a failure of the double-acting hydraulic cylinder, the cylinder can be easily removed and the locking system can be used manually, as it is in the current state.

The disadvantage of this design is the need to install a new pressure line to control the hydraulic cylinder, or to use hydraulic hoses that are already mounted on the boom, but serve to control the implement.

After the completion of the structural design, the FEM analysis was performed using the ANSYS program [11-13]. The results of the analysis are shown in Fig. 13. The analysis was performed on a design with a double-acting hydraulic cylinder. Unnecessary components of the analysis model that do not have a significant effect on the results were removed from the design.

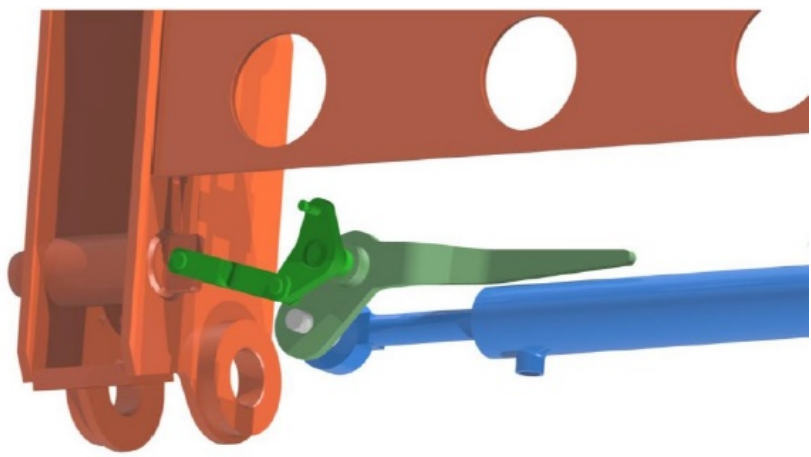

Fig. 12. Part of the lever transmission design

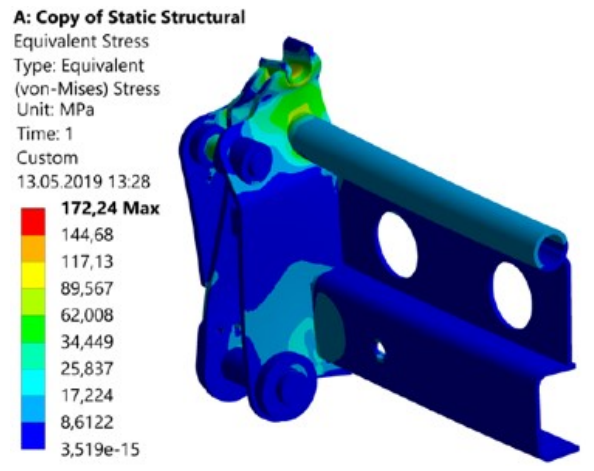

Fig. 13. Distribution of the equivalent von Misses stress in the most loaded area of the whole design

After this step, the basic carrier parts of the quick clamping device remained. There was applied the load. The quick clamping device is attached to the boom with four pins that are inserted into the holes in the side frames. For this reason, the axes of these holes are set as boundary conditions and the quick clamping device is fixed there. Another boundary condition is the applied force. This force simulates the load that occurs when the bucket is fully loaded and passes through the center of the bucket. Because of the complexity of the issue, the authors decided to include in this paper only the stress distribution gained from the simulation. The detailed description will be addressed in further issue solving.

As a part of future research, a multibody model of the skid-steer loader including the modified part will be created. It will serve for investigation of dynamic properties of the skid-steer loader in operation conditions. Dynamic loads of the newly designed quick clamping device will be evaluated in order to identify its properties in terms of long-term operation [14-17].

\section{Conclusions}

1. The paper presents a methodology for designing an optimized quick clamping device design for attachments of a skid steer loader.

2. The article is an important summary of information that is necessary for processing of a suitable design and also necessary to realize due to the wide scope of the mentioned issue.

3. This section should only contain the main conclusions based on the present research.

4. An essential feature of such a processed problem is that the implementation of this technical solution in the loader will increase the comfort of the machine operator, because there is no need to exit, manually manipulate and re-enter into the working machine. 
5. The requirement of maintaining the main dimensions of the quick clamping device design has been met, i.e., all the main objectives can be considered to be met.

\section{Acknowledgements}

This work was supported by the Cultural and Educational Grant Agency of the Ministry of Education of the Slovak Republic in the project No. KEGA 023ŽU-4/2020: Development of advanced virtual models for studying and investigation of transport means operation characteristics.

\section{References}

[1] Aquilera-Marinovic S., Torres-Torriti M, Auat-Cheein F. General dynamic model for skid-steer mobile manipulator with wheel-ground interactions. IEEE-ASME Transactions on Mechatronics, vol. 22, 2017, pp. 433-444.

[2] Dabrowska A., Jaskolowski M., Konopka S., Prybysz M. Stability research of lightweight sixwheeled ground vehicles. Proceedings of "Intelligent Technologies in Logistics and Mechatronics Systems-ITELMS 2015", May 21-22, 2015, Panevezys, Lithuania, pp. 83-89.

[3] Ren F., Liu XH., Chen JS., Zeng P., Jia XF. Analysis of Skid Steer Loader Steering Characteristic. Advances in Mechanical Engineering, vol. 7, 2015, 9 pages.

[4] Rubenstone J. Kubota introduces skid steerto fill out compact equipment line. ENR (Engineering News-Record), vol. 274, 2015.

[5] Yengst CR. A lot of questions about growth prospects in skid-steer loader markets. Diesel Progress North American Edition, vol. 68, 2002, p. 4.

[6] Mercer M. Multiple control options for newest Hydra-Mac skid-steer loader. Diesel Progress North American Edition, vol. 63, 1997, pp. 26-26.

[7] Dveges R., Durovsky F., Fedak V. Skid steering of robotic vehicle for autonomous applications. Proceedings of $20^{\text {th }}$ International Confernce on "Electrical Drives and Power Electronics", September 24-26, 2019, pp. 335-341.

[8] Gelati R., Reina G. Terrain awareness using a tracked skid-steering vehicle with passive independent suspensions. Frontiers Robotics Al, vol. 6, 2019.

[9] Zhang J., Chen S. Modelling and study of active vibration control for off-road vehicle. Vehicle System Dynamics, vol. 52, 2014, pp. 581-607.

[10] Lopatka M.J., Muszynski T. Determining of drivetrain system skid steer 6x6 wheeled robot load. Proceedings of $30^{\text {th }}$ International Symposium on "Automation and Robotics in Construction and Mining", August 11-13, 2013, Montreal, Canada.

[11] Khomichev A., Volkov A. Chassis design of skid steer loaders. Proceedings of International Conference on "Modern Trends in Manufacturing Technologies", September 9-13, 2019, Sevastopol, Russian Federation.

[12]Fomin O., Gerlici J., Lovksa A., Kravchenko K., Potapenko P., Fomina A., Hauser V. Durability determination of the bearing structure of an open freight wagon body made of round pipes during its transportation on the railway ferry. Communications - Scientific Letters of the University of Zilina, vol. 21, 2019, 28-34.

[13] Stastniak P., Moravcik M., Baran P., Smetanka L. Computer aided structural analysis of newly developed railway bogie frame. MATEC Web of Conference, vol. 157.

[14] Lack T., Gerlici J. The assessment of the integration methods for the rail vehicle ride dynamics solution. MATEC Web of Conferences, vol. 157.

[15] Lack T., Gerlici J. Integration methods for rail vehicle ride dynamics solution assessment. Proceedings of " $23^{\text {rd }}$ Conference: Current Problems in Rail Vehicles 2017", September 20-22, 2017, Ceska Trebova, Czech Republic.

[16] Hauser V., Nozhenko O., Kravchenko K., Loulova M., Gerlici J., Lack T. Impact of three axle boxes bogie to the tram behavior when passing curved track. Procedia Engineering, vol. 192, 2017, pp. 295-300.

[17] Gerlici J., Sakhno V., Yefymenko A., Verbitskii V., Kravchenko A., Kravchenko K. The stability analysis of two-wheeled vehicle model. MATEC Web of Conferences, vol. 157. 